\title{
ARTICLE Nicotine dependence (trait) and acute nicotinic stimulation (state) modulate attention but not inhibitory control: converging fMRI evidence from Go-Nogo and Flanker tasks
}

\author{
E. Lesage $\mathbb{D}^{1,2}$, M. T. Sutherland $d^{1,3}$, T. J. Ross (iD) ${ }^{1}$, B. J. Salmeron (iD) ${ }^{1}$ and E. A. Stein (iD)
}

Cognitive deficits during nicotine withdrawal may contribute to smoking relapse. However, interacting effects of chronic nicotine dependence and acute nicotine withdrawal on cognitive control are poorly understood. Here we examine the effects of nicotine dependence (trait; smokers $(n=24)$ vs. non-smoking controls; $n=20)$ and acute nicotinic stimulation (state; administration of nicotine and varenicline, two FDA-approved smoking cessation aids, during abstinence), on two well-established tests of inhibitory control, the Go-Nogo task and the Flanker task, during fMRI scanning. We compared performance and neural responses between these four pharmacological manipulations in a double-blind, placebo-controlled crossover design. As expected, performance in both tasks was modulated by nicotine dependence, abstinence, and pharmacological manipulation. However, effects were driven entirely by conditions that required less inhibitory control. When demand for inhibitory control was high, abstinent smokers showed no deficits. By contrast, acutely abstinent smokers showed performance deficits in easier conditions and missed more trials. Go-Nogo fMRI results showed decreased inhibition-related neural activity in right anterior insula and right putamen in smokers and decreased dorsal anterior cingulate cortex activity on nicotine across groups. No effects were found on inhibition-related activity during the Flanker task or on error-related activity in either task. Given robust nicotinic effects on physiology and behavioral deficits in attention, we are confident that pharmacological manipulations were effective. Thus findings fit a recent proposal that abstinent smokers show decreased ability to divert cognitive resources at low or intermediate cognitive demand, while performance at high cognitive demand remains relatively unaffected, suggesting a primary attentional deficit during acute abstinence.

Neuropsychopharmacology (2020) 45:857-865; https://doi.org/10.1038/s41386-020-0623-1

\section{INTRODUCTION}

Tobacco addiction remains the leading cause of preventable death in the world [1]. The vast majority of smokers express a strong desire to quit, but most quit attempts fail within a week [2]. An important cause of relapse is the nicotine-withdrawal syndrome (NWS), which is characterized by affective $[2,3]$, reward [4-6], and cognitive deficits $[7,8]$. Cognitive control deficits have been hypothesized to be a key symptom of drug withdrawal $[9,10]$, both because they impact normal daily functioning and because they induce a deficit state making it more difficult to remain abstinent.

Nicotine is a nonselective nicotinic acetylcholine receptor (nAchR) agonist, acting on multiple neurotransmitter systems, including cholinergic, dopamine (DA), norepinephrine (NE), glutamate, and GABA [11]. Nicotine is an indirect DA agonist, binding to a $4 \beta 2 \mathrm{nArchRs}$ on midbrain DA neurons and stimulating the mesocorticolimbic (MCL) system, which consists of striatal, limbic, and prefrontal terminals of these midbrain DA neurons [12]. Nicotine's actions on different neurotransmitter systems can interact with each other. For example, the NE system targets overlapping prefrontal circuitry to the MCL system and modulates attention [13], while nicotine's glutaminergic effects modulate descending corticostriatal pathways [14]. Nicotine's reinforcing properties derive from its action upon the dopaminergic $\mathrm{MCL}$ system. Dependence is thought to develop through chronic stimulation of this system, leading to neuroplastic changes in $\mathrm{MCL}$ circuitry that downregulate DA levels $[10,15]$. This hypodopaminergic withdrawal state that characterizes drug dependence is one prominent mechanistic hypothesis through which abstinence from nicotine could impair smokers' cognitive control.

In line with this mechanistic hypothesis, effective pharmacological smoking cessation aids such as nicotine replacement therapy and varenicline (Chantix ${ }^{\circledR}$ ) predominantly target $\alpha 4 \beta 2$ nAchRs. The latter serves as a partial agonist at these receptors, acting as a weak agonist in the absence of nicotine, and as a partial antagonist in the presence of nicotine [16]. The in vitro receptor-binding mechanisms of these drugs and their efficacy at a clinical level are established [17-19], but it is less clear how their systems-level neurobiological mechanisms affect the cognitive and affective deficits seen in the human NWS and how this differs from these drugs' effects in non-smokers. Nicotine and varenicline are administered clinically in both the nicotine-sated and in the acute and prolonged abstinent state. How their neurobiological mechanisms of action are biased under these

\footnotetext{
${ }^{1}$ Neuroimaging Research Branch, National Institute on Drug Abuse, Intramural Research Program, National Institutes of Health, Baltimore, MD, USA Correspondence: E A. Stein (Estein@nih.gov)

${ }^{2}$ Present address: Department of Experimental Psychology, Gent University, Ghent, Belgium

${ }^{3}$ Present address: Department of Psychology, Florida International University, Miami, FL, USA
}

Received: 21 August 2019 Revised: 29 December 2019 Accepted: 17 January 2020

Published online: 29 January 2020 
disease cycles and how that might affect clinical efficacy is poorly understood. To this end, we investigated the effects of acute nicotinic receptor stimulation through a nicotine patch and varenicline pill interaction on inhibitory control mechanisms in both smokers and non-smoking control participants.

We used two tasks to probe inhibitory control and error monitoring: the Go-Nogo task and the Eriksen Flanker task [20]. These have previously characterized cognitive control deficits in substance abuse, including cocaine, alcohol, and nicotine [21, 22]. Both tasks tax inhibitory control over a dominant response tendency and largely recruit the same neural networks [23-25]. The Go-Nogo task requires the inhibition of a prepotent motor response (especially when Nogo trials are rare, as in this study), while in the Flanker task response dominance elicited by an irrelevant task dimension must be suppressed [25].

Neuroimaging studies in substance-dependent populations have consistently reported hypoactivation in frontal cortical areas associated with cognitive control [21], notably the dorsal anterior cingulate cortex (dACC), dorsolateral prefrontal cortex (DLPFC), and right inferior frontal gyrus (IFG). Administration of a DA antagonist decreases neural activity in these regions and cognitive control performance in both smokers and non-smokers [26]. This hypofrontality is consistent with the DA-deficiency model of addiction [27] and generalizes to other substance use disorders, including cocaine addiction [28].

Behavioral evidence for inhibitory control deficits in nicotinedependent populations is mixed, with some studies reporting deficits [29, 30], while others do not [31, 32]. The inconsistency in the literature may be partially attributable to the drug state of the cohorts used; most studies compared nicotine-sated smokers with non-smokers while others required smokers to be abstinent $[22,29,30]$. In non-smoker populations, nicotine enhances cognitive and attentional performance [33-36], with evidence for inhibitory control effects less clear [37]. To the best of our knowledge, no study has directly examined the effects of nicotine dependence on inhibitory control behavior and neural mechanisms in both the nicotine-withdrawal and nicotine-sated conditions, even though these comparisons are crucial to understanding the NWS and its role in treatment success or, more often, relapse.

The current study overcomes these limitations by investigating both smoking state (i.e., 12-h abstinent vs. acutely nicotine sated) and smoking trait (smokers vs. non-smokers) effects. We examined the effects of nicotine patch and varenicline pill (both alone and in combination to mimic how these drugs are used clinically) using a Go-Nogo and a Flanker task as probes of inhibitory control. Both smokers and non-smokers performed these tasks during functional magnetic resonance imaging (fMRI) acquisition; the 12-hour abstinent manipulation in smokers was intended to mimic the first day of a quit attempt, which is when smokers are most vulnerable to relapse [38]. We hypothesize that abstinent smokers will perform worse than non-smokers on these tasks, specifically in task conditions that require higher inhibitory control, and that this deficit will be alleviated, at least in part, by nicotine and, to a lesser extent, varenicline. In addition, we expect that neural responses during successful and failed inhibition will be affected by chronic nicotine-dependence trait and acute nicotinic stimulation in a manner reflecting their effects on performance.

\section{METHODS}

\section{Participants}

Participants were 24 smokers ( 12 females) and 20 non-smokers (10 females). Written informed consent was obtained from the NIDAIRP Institutional Review Board. Participants were right-handed, between the ages of 18 and 55 years, and had no reported history of neurological or psychiatric disorders or current or past substance dependence (other than nicotine in smokers). Nonsmokers reported no history of daily nicotine use and no smoking within the past 2 years. Groups were matched on gender and ethnicity (Table S1). Smokers were older than controls $\left(t_{41.32}=\right.$ $2.12, p<0.05)$, and age was therefore included as a covariate in all between-group analyses. Data from one male non-smoker were excluded due to consistently poor behavioral performance and excessive head motion.

\section{Study design}

Participants underwent six scanning sessions as part of a fully counterbalanced, two-drug, double-blind, placebo-controlled study (see Fig. 1). Results from the four completely counterbalanced sessions are reported herein. The reported data are part of a larger study that also probed effects of nicotine and varenicline on reward anticipation [4], economic decisionmaking [39], and emotional reactivity [3, 40]. For detailed information on the experimental design and MRI acquisition parameters, see Supplementary Materials and methods.

\section{Go-Nogo and Flanker tasks}

Participants carried out four 5-min blocks (300 trials each) of a Go-Nogo task with alternating " $X$ " and " $Y$ " stimuli [24]. Participants were instructed to respond when the presented stimulus (" $X$ " or " $Y$ ") was different from the previously presented stimulus (e.g., $X-Y$; Go trial) and suppress this prepotent response when the stimulus was identical to the previous stimulus (e.g., $\mathrm{X}-\mathrm{X}$; No-go trial; Fig. 2a). The infrequent Nogo trials (100 out of 1200 trials; $8.3 \%$ ) were temporally jittered to optimally estimate the bloodoxygen-level-dependent (BOLD) response. Participants also performed four 9-min 130-trial runs of a speeded version of the Flanker task. Participants were instructed to respond to a target stimulus, an " $\mathrm{H}$ " or an " $\mathrm{S}$ " with a button press. Flanker items (HH_HH or SS_SS) were presented, which matched (congruent trials, 50\%) or did not match (incongruent trials, 50\%) the target

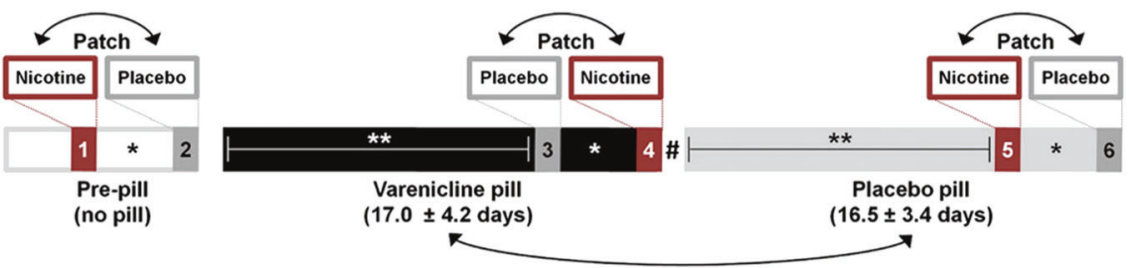

Fig. 1 Study design. All participants completed 6 scanning sessions; the current study reports data from the four completely counterbalanced sessions (sessions 3-6). At three points during a varenicline administration regime, (i.e., pre-pill, 2 weeks varenicline pill, 2 weeks placebo pill), participants were scanned twice, once wearing a nicotine patch and once wearing a placebo patch. Non-smokers received a 7-mg nicotine patch dose, while smokers received a dose that matched daily nicotine intake $(21,28,35$, or $42 \mathrm{mg}$ patches for $10-15$, 16-20, 21-25, and >25 cigarettes/day, respectively). Smokers were $12 \mathrm{~h}$ abstinent from nicotine (verified through CO levels). ${ }^{* *} V$ Varenicline and placebo pill sessions were separated by $>2$ weeks. *Nicotine and placebo patch scans were separated by an average of $2.9 \pm 1.7$ days. \#No washout interval separated medication periods. Double-headed arrows indicate randomized and counterbalanced order of the sessions. Figure reproduced with permission from Sutherland et al. [49]. 
A. Go-Nogo task

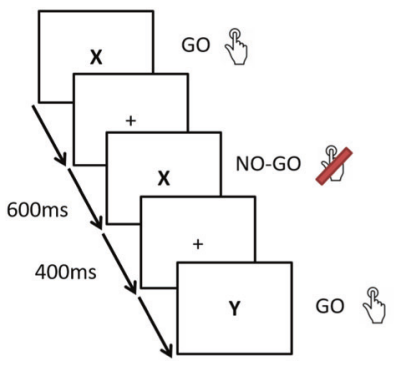

B. Commission errors (Nogo trials)

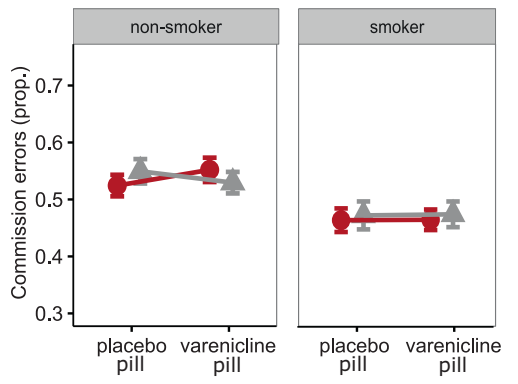

C. Omission errrors (Go trials)

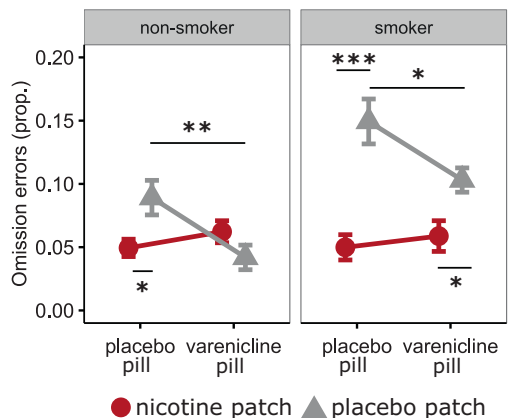

Fig. 2 Go-Nogo task. a Trial timing of the task. Stimuli alternated between " $X$ " and " $Y$ " on each trial; participants were instructed to respond when the stimulus presented on the screen was different from the previously presented stimulus (Go trial) and to suppress a response when the stimulus was identical to the previously presented stimulus (No-go trial). Trials were spaced $1 \mathrm{~s}$ apart. Nogo trials are modeled against the implicit baseline, which consists of Go trials and fixation between trials (as in $[26,28,60,61])$. The timing of Nogo trials is temporally jittered so that these trials can be modeled. b, c Go-Nogo performance. Error rate on Nogo (b) and Go (c) trials. Error bars indicate $+/-1$ standard error of the mean. ${ }^{*} p<0.05,{ }^{* *} p<0.01,{ }^{* * *} p<0.001$.

(Fig. 3a). See Supplementary Materials and methods for more detailed information.

Behavioral analyses

Analyses were performed in $\mathrm{R}$ (www.r-project.org/) using packages afex and phia. For the Go-Nogo task, we performed mixed-model analyses of variance (ANOVAs) on two dependent variables: the error rate on Nogo trials (commission errors, indicating a failure of response inhibition), and the error rate on Go trials (omission errors, indicating attention lapses). For the Flanker task, we performed mixed-model ANOVAs on four dependent variables: the error rate on congruent and on incongruent trials (indicating a failure to overcome a prepotent response) and the missed trial rate on both trial types (the latter three indicating a lack of attention). We compared performance on incongruent and congruent trials across sessions as a manipulation check. Independent variables were the betweensubject variable GROUP (smokers vs. non-smokers) and the withinsubject variables NICOTINE (nicotine patch vs. placebo patch) and VARENICLINE (varenicline pill vs. placebo pill), and age as a covariate. Interactions were followed up with within-group ANOVAs using NICOTINE and VARENICLINE as independent variables.

\section{Imaging analyses}

Preprocessing. Imaging analyses were carried out in AFNI [41]. Functional scans were preprocessed using standard methods: slice-time correction, image alignment to MPRAGE, motion correction, spatial registration to the Talairach template, and smoothed to $8 \mathrm{~mm}$ full width at half maximum.

Single-subject analysis. Three Go-Nogo regressors of interest were modeled against the implicit baseline: correctly executed Nogo trials, incorrectly executed Nogo trials, and incorrectly executed Go Trials. Correctly executed Go trials were included into the implicit baseline. Two contrasts of interest were calculated: INHIBITION [Nogo Correct - implicit baseline] and ERROR [Nogo Incorrect - Nogo Correct]. Four Flanker regressors of interest were modeled against the implicit baseline: correct and incorrect responses for congruent and incongruent trials. Two contrasts of interest were estimated: INHIBITION [Incongruent Correct Congruent Correct] and ERROR [Incongruent Incorrect - Incongruent Correct]. We excluded (censored) timepoints where Euclidian displacement between successive frames exceeded 0.3 $\mathrm{mm}$ or where the DVARS [42] exceeded 1.3. For the Flanker task, missed responses were modeled separately as a regressor of no interest. For the Go-Nogo task, timepoints where responses were absent for $\geq 10$ trials were similarly modeled separately. First-level design matrices for both tasks included the regressors of interest and their temporal derivative, polynomials for each block, and six regressors that modeled head motion.

Group-level analysis. Average activity patterns for INHIBITION and ERROR contrasts were computed with $t$ tests over subjects' beta weight maps (averaged over sessions). Results were whole-brain familywise error (FWE) corrected (alpha $<0.05$, voxel-wise $p<$ 0.001 , cluster size 22 voxels, determined with a 3dClustSim algorithm).

Group and drug effects for both contrasts were examined with mixed ANOVAs (between-subject factor GROUP, within-subject factors NICOTINE and VARENICLINE, age as covariate). Significant GROUP interactions were followed by within-group analyses using NICOTINE and VARENICLINE as factors. We constructed a functional small-volume mask of interest, derived separately for each task from an OR mask of the INHIBITION and the ERROR contrasts (see Supplementary Materials and methods). Results within this volume of interest were FWE corrected (alpha $<0.05$, voxel-wise $p<0.01$, cluster size 24 voxels (Go-Nogo) or 22 voxels (Flanker), determined with a 3dClustSim algorithm).

\section{RESULTS}

Behavioral results

Go-Nogo performance. The rate of commission errors, the failure to suppress a prepotent response, was unaffected by GROUP, NICOTINE, VARENICLINE, or their interactions (Fig. 2b). By contrast, omission errors, missed responses on Go trials, showed significant NICOTINE $\quad(F(1,123)=33.72, \quad p<0.001), \quad$ NICOTINE $\times$ GROUP $(F(1,123)=6.22, \quad p=0.014), \quad$ and NICOTINE $\times$ VARENICLINE $(F(1,123)=11.55, p<0.001)$ effects. Specifically, smokers in the acute abstinence (placebo patch) condition committed many more omission errors than under the nicotine patch condition (NICOTINE: $F(1,69)=30.23, p<0.001$ ), and this nicotinic effect was blunted by the presence of varenicline (NICOTINE $\times$ VARENICLINE: $F(1,69)=4.65, p=0.034)$ in accordance to the drug's partial antagonist role [16]. A smaller but qualitatively similar pattern is observed in non-smokers, where omission rates were lower on NICOTINE $(F(1,54)=7.80, p=0.007)$, except when varenicline was also administered (NICOTINE $\times$ VARENICLINE: $F(1,54)=8.90, p=$ 0.004 ; Fig. 2c). Smokers tended to be faster on Go trials ( $F$ $(1,61.7)=3.00, p=0.088)$ and were faster when making commission errors $(F(1,67.7)=4.86, p=0.031)$. None of the pharmacological manipulations affected reaction time (RT; see Supplementary Materials and methods). Intelligence quotient (IQ), gender, or 
A. Flanker task

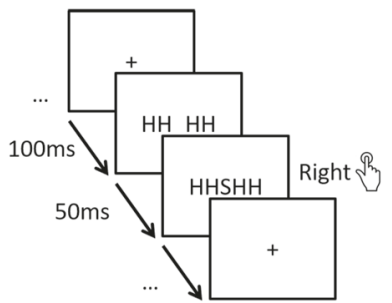

B. Performance across sessions

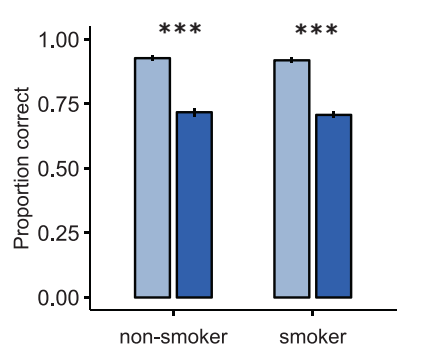

$\square$ congruent $\square$ incongruent
C. Errors: incongruent condition
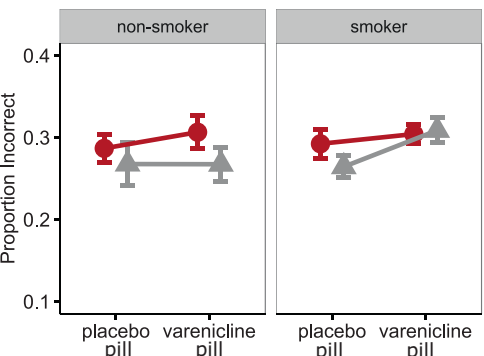

D. Missed trials: incongruent condition

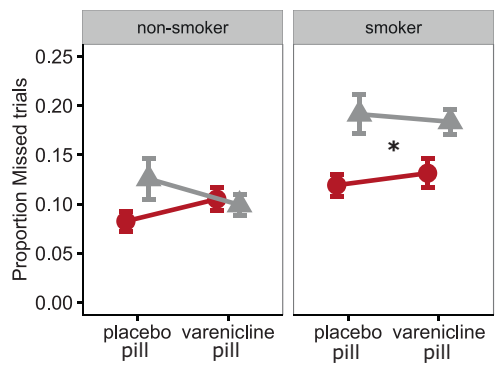

E. Errors: congruent condition

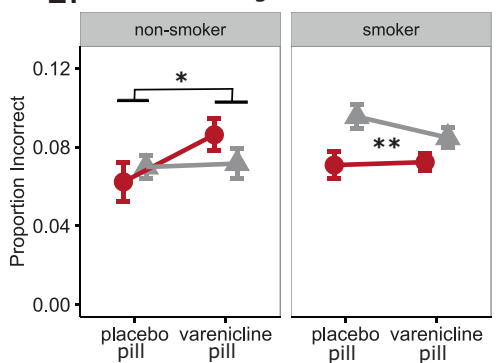

F. Missed trials: congruent condition

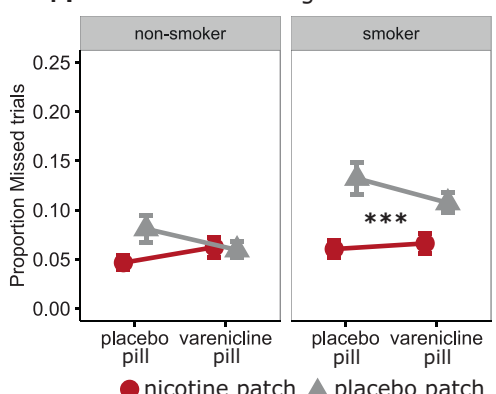

Fig. 3 Flanker task. a Trial timing of the task. At the start of each trial, prior to presentation of the target stimulus, flanker items (HH_HH or SS_SS) were presented. After $100 \mathrm{~ms}$, the center target stimulus was added to the array and both remained on the screen for $50 \mathrm{~ms}$. The response deadline was individualized to ensure an adequate difficulty level and error rate [40]. The design was event related, allowing estimation of the BOLD response associated with each trial type. Ellipses indicate variable temporal jitter, enabling statistical modeling of each trial against the implicit baseline consisting of fixation periods between trials. b-f Behavioral performance. b Performance across sessions. c, d Performance on Incongruent trials. e, f Performance on congruent trials. For analyses with reaction time as the dependent variable, please see Fig. S5. Error bars indicate $+/-1$ standard error of the mean. ${ }^{*} p<0.05,{ }^{* *} p<0.01,{ }^{* *} p<0.001$.

session order did not significantly affect dependent measures (see Supplementary Materials and Methods).

Flanker performance. Both groups performed better (smokers: $F(1,161)=77.18, p<0.001$; non-smokers: $F(1,126)=56.11, p<$ 0.001 ) and faster (smokers: $F(1,161)=78.69, p<0.001$; nonsmokers: $F(1,126)=32.04, \mathrm{p}<0.001)$ in the congruent than in the incongruent condition, with no group difference across sessions (CONDITION $\times$ GROUP, Accuracy: $F(1,287)=0.006, p=$ 0.939; RT: $F(1,287)=0.08, p=0.778$, Figs. $3 \mathrm{~b}$ and S6A). Examining accuracy per condition, no main or interaction effects of GROUP, NICOTINE, or VARENICLINE were found in the incongruent condition (Fig. 3c). By contrast, in the congruent condition, a main effect of NICOTINE $(F(1,123)=5.68, p=0.018)$ and trends for VARENICLINE $(F(1,123)=3.55, p=0.062)$, GROUP $\times$ VARENICLINE $(F(1,123)=2.77, p=0.099)$, and NICOTINE $\times$ VARENICLINE $(F(1,123)=$ 3.24, $p=0.074$ ) were identified (Fig. 3e). These patterns were driven by worse performance in smokers without nicotine $(F(1,69)$ $=9.49, p=0.003)$ and worse performance with varenicline in the non-smoker group $(F(1,54)=4.46, p=0.039)$. Both groups missed more trials in the incongruent than in the congruent conditions $(F(1,287)=11.45, p<0.001)$, missed fewer trials with the nicotine patch $(F(1,287)=31.20, p<0.001)$, with this nicotinic effect smaller in the presence of varenicline pill (NICOTINE $\times$ VARENICLINE: $F(1,287)=5.95, p=0.015)$ and tended to be larger in smokers (NICOTINE $\times$ GROUP: $F(1,287)=2.80, p=0.099$; Fig. 3d, f). These patterns were explained by a strong NICOTINE main effect in smokers $(F(1,69)=19.10, p<0.001)$ and a weaker NICOTINE effect in non-smokers $(F(1,54)=5.84, p=0.019)$. IQ, gender, or session order did not significantly affect dependent measures (see Supplementary Materials and Methods).

In sum, for both cognitive control tasks, we failed to find effects of smoker (GROUP) or acute nicotinic stimulation (NICOTINE or VARENICLINE) in conditions that require inhibitory control (Nogo condition and Incongruent condition). In contrast, we do find robust DRUG and smoker (GROUP) effects in the control conditions, which require attention but no response inhibition, as well as in the rate of missed responses. This suggests that acute nicotine withdrawal impairs attentional processes rather than inhibitory control and that this impairment is ameliorated by nicotinic stimulation.

Neuroimaging results

Go-Nogo task. The INHIBITION task map (Fig. 4a; Table S5) shows robust activity in the salience network [43], encompassing bilateral IFG, anterior insula (AI), and $\mathrm{dACC}$, stretching out into presupplementary motor areas (pre-SMA), as well as the striatum and thalamus. Smaller areas in medial frontal cortex and precuneus showed less activation during Nogo trials than during Go trials. These patterns are in line with meta-analysis of Go-Nogo tasks [24]. In the ERROR contrast (Fig. 4b), regions of the dACC and preSMA, left dorsal striatum, middle frontal gyrus, and cuneus showed increased BOLD activation during commission errors compared to correctly withholding a response.

Effects of GROUP, NICOTINE, and VARENICLINE were assessed with mixed ANOVA for both contrasts, FWE corrected within the task mask of interest (Fig. 5a, b). For the INHIBITION contrast, there was a GROUP effect in the right $\mathrm{Al}$ and right putamen: smokers showed less activity than non-smoking controls (Fig. 5a). In addition, there was a main effect of NICOTINE within a region on the border of dACC and pre-SMA that was less active under the nicotine patch compared to the placebo patch condition (Fig. 5b). No session effects were found for the ERROR contrast.

Flanker task. The INHIBITION contrast (across all sessions) revealed increased activation during correct incongruent trials compared to correct congruent trials in bilateral Al and ACC/preSMA (nodes of the salience network [43], as well as bilateral DLPFC and left superior parietal cortex (Fig. 4c, Table S6), consistent with a previous meta-analysis of Flanker tasks [44]. In contrast, the right 
A. Inhibition: Go-Nogo Nogo Correct

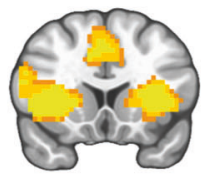

$\mathrm{Y}=-16.5$

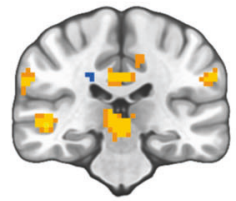

R $Y=26.5$
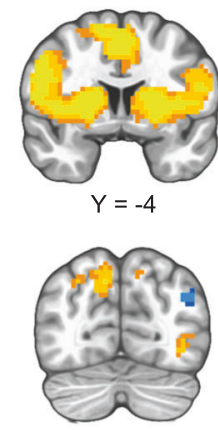

$\mathrm{Y}=70$
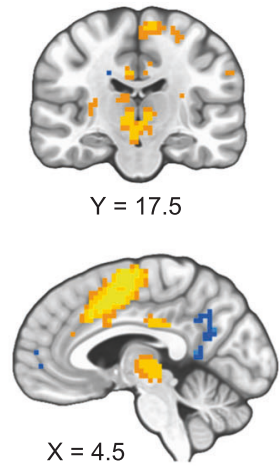

displayed threshold $p<10^{-6}$

C. Inhibition: Flanker

Incongruent Correct - Congruent Correct

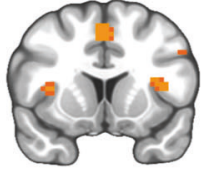

$\mathrm{Y}=-16.5$

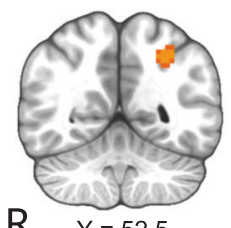

R $Y=52.5$

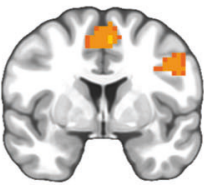

$Y=-1.5$

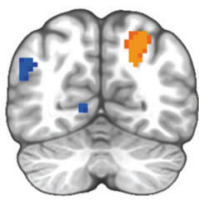

$\mathrm{Y}=61.5$

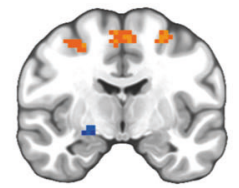

$\mathrm{Y}=7.5$

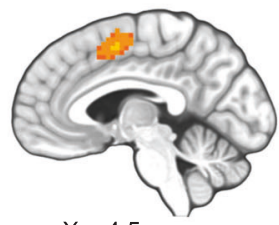

$X=4.5$
B. Error: Go-Nogo

Nogo Incorrect - Nogo Correct

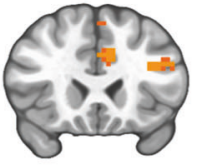

$\mathrm{Y}=-22.5$

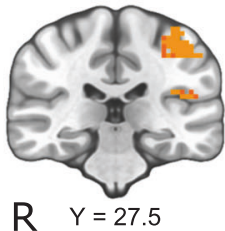

R $Y=27.5$

displayed threshold $p<10^{-3}$
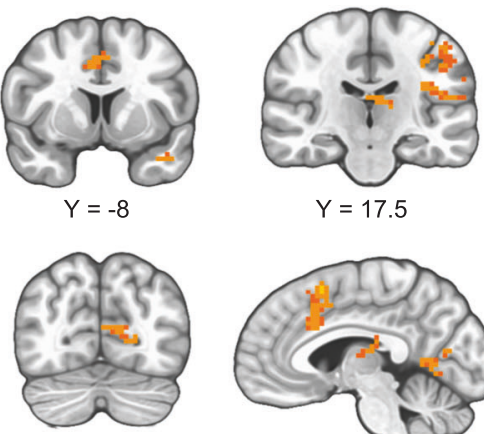

$Y=70$ 3
D. Error: Flanker

Incongruent Incorrect - Incongruent Correct

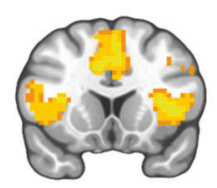

$Y=-15.5$

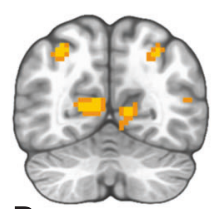

R $Y=60$

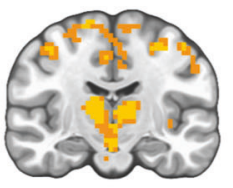

$\mathrm{Y}=13.5$

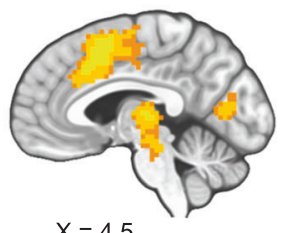

$x=4.5$ displayed threshold $p<10^{-6}$

Fig. 4 Task maps for the Go-Nogo and Flanker contrasts. a Brain areas with greater (orange) or smaller (blue) response to successfully inhibiting a prepotent response compared to baseline (correctly executed Go trials). b Brain areas that showed greater (orange) response to failed inhibitions than to successful inhibitions on Nogo trials. c Brain areas with more (orange) or less (blue) activity in response to correctly executed incongruent trials than to correctly executed congruent trials. d Brain areas showing greater activity during errors on congruent trials than during the correct execution of incongruent trials. Results were whole-brain FWE corrected (alpha $<0.05$, voxel-wise $p<0.001$, cluster size 22 voxels. Owing to large task effect size, a and $\mathbf{d}$ are displayed with a more stringent threshold for display purposes, see Figs. S3 and S4 for images thresholded at $p<0.001)$. Radiological orientation: right is presented on the left.

inferior parietal cortex, left occipital cortex, and left thalamus showed greater response in congruent compared to incongruent conditions. The ERROR contrast identified a robustly activated network, including bilateral Al, dACC/pre-SMA and SMA, bilateral thalamus, DLPFC, superior parietal lobule, and occipital cortex (Fig. 4d). The mixed ANOVA examining the effects of GROUP, NICOTINE, and VARENICLINE within these masks did not yield any significant session effects for either task contrast.

\section{DISCUSSION}

NWS, characterized by dysregulated affective and cognitive processing, has been hypothesized to be the principal factor in smoking cessation failures. As such, a better understanding of the neurobiological mechanisms underlying the NWS and how pharmacotherapies might help alleviate specific withdrawal signs linked to relapse is critically needed. In this study, we hypothesized that acute nicotine withdrawal would disrupt inhibitory control and that this deficit would be reversed in the presence of two commonly used pharmacotherapeutic agents: nicotine patch and/or varenicline pill. We employed two cognitive control tasks to test this hypothesis: the Go-Nogo and the Flanker task.
Contrary to our hypothesis, we did not find effects on performance in demanding conditions requiring inhibitory control of either task during abstinence. However, we did find the hypothesized drug and condition (abstinence vs. satiety) effects on measures of attention and on the number of missed trials (i.e. omissions) in both tasks. That is, our behavioral results indicate deficits in sustained attention in abstinent, but not in sated, smokers, which are alleviated with nicotinic receptor stimulation. Non-smokers exhibited more subtle beneficial effects of nicotinic stimulation on omissions in the Go-Nogo task and missed trials in the Flanker task. Imaging analyses during the successful withholding of an inappropriate response on Go-Nogo trials showed lower right $\mathrm{Al}$ and putamen activity in smokers independent of state and decreased $\mathrm{dACC} /$ pre-SMA activity in the presence of a nicotine patch.

Our results show that nicotine-dependence trait and nicotinic stimulation effects (state) were present in conditions that did not require response inhibition but did require attention. Notably, patterns were strikingly similar across the two tasks, adding confidence to this somewhat counter-intuitive finding. In the Go-Nogo task, abstinent smokers failed to execute responses when they should have, i.e., errors of omission. In the Flanker task, 


\section{A. Go-Nogo Inhibition Smokers < Non-smokers}
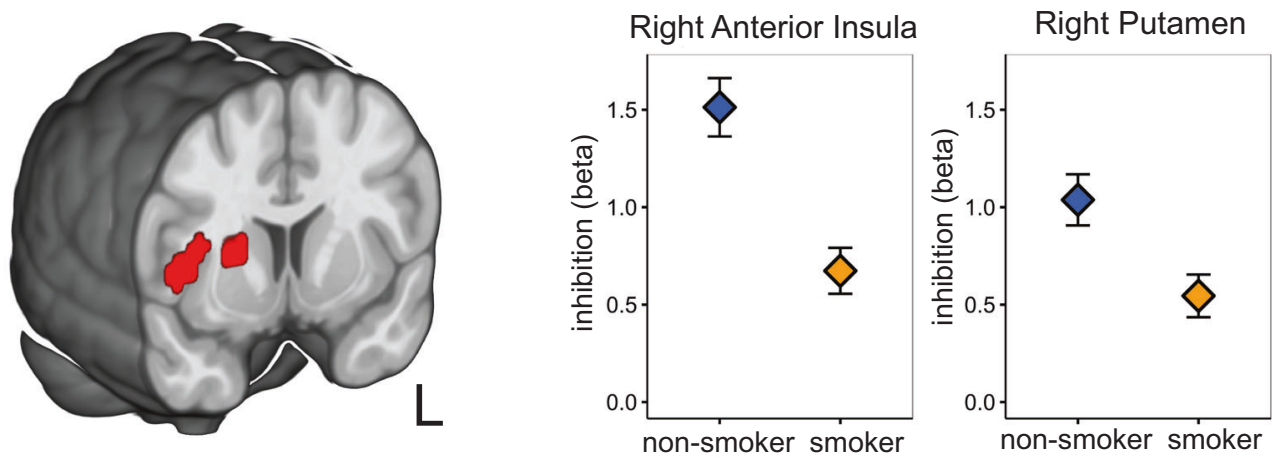

\section{B. Go-Nogo Inhibition Nicotine < Placebo}
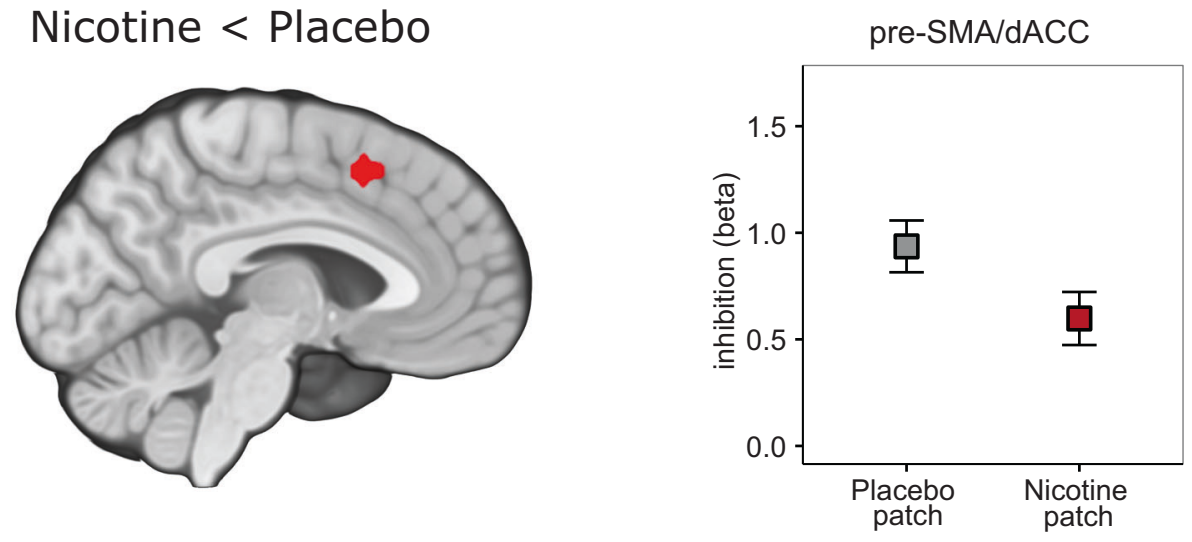

Fig. 5 Group and Nicotine effects on neural activity during the Go-Nogo task. a Smokers show hypoactivation of right anterior insula and right putamen during correct inhibition of a prepotent response compared to non-smokers. b Across groups, nicotine lowered inhibitionrelated activity in the superior frontal gyrus (pre-SMA/dACC). Both panels: Activations of FWE corrected within the mask of interest (alpha < 0.05 , voxel-wise $p<0.01$, cluster size 24 voxels). Extracted regression weights and error bars (SEM) presented to aid interpretation only; no statistical inference should be drawn. Radiological orientation: right is presented on the left.

12-h nicotine-deprived smokers were more likely to fail to press the correct button when flankers primed the correct location. Moreover, missed responses were more prominent in abstinent smokers for both congruent and incongruent conditions, with this deficit marginally stronger in the congruent (less demanding) condition. In all cases, these deficits were alleviated with the administration of nicotine, following which performance was similar to that of non-smokers. Varenicline, a partial agonist at the a4 $\beta 2$ nicotinic receptors, significantly improved performance only in the Go-Nogo task. Generalizing across tasks, nicotinic receptor stimulation improved sustained attention in both smokers and non-smokers. Effects were greater in smokers, who-when abstinent-appeared to fail to allocate appropriate attentional resources at low levels of cognitive demand. Rather than a blanket inability to engage cognitive and attentional resources, the deficit appears specific to conditions where only relatively minimal attention is required. The robust effects on attention that we report are in line with literature on attention-enhancing effects of nicotine $[13,45]$ and problems with sustained attention in smokers [37, 46-48].

In contrast, we did not find behavioral effects in task conditions requiring high inhibitory control. While it is possible that such effects exist but were undetectable in this sample, this seems unlikely given the presence of clear drug effects at the physiological level and in the control conditions, the reproducibility using two independent, well-characterized tasks, and effects on other tasks performed in the same cohort and in the same session, including reward processing, emotional processing, and reversal learning [3, 4, 39, 49]. We are therefore confident that the imposed pharmacological manipulations exerted appropriate pharmacodynamic effects. Moreover, the absence of inhibitory control deficits is not exceptional in the literature. Evidence for effects of nicotine dependence (trait) $[29,30,32,50-52]$, as well as nicotine satiety within subject (state) in inhibitory control $[37,47]$ is mixed.

One way to interpret our results is to consider the varying demand hypothesis proposed by Fedota et al. [53]. Using a Flanker task with three levels of difficulty, the authors found that smokers and non-smokers' performance and brain activity only differed at intermediate levels of difficulty. Similarly, work using a smoking Nback task with three levels of difficulty showed the most bias processing at intermediate level of demand [54]. Thus, rather than a generalized reduction in cognitive capacity, it may be that abstinent smokers' deficits lie in the inability to allocate cognitive and attentional resources in response to slightly increased demand. That is, when cognitive or inhibitory demand is clearly high, abstinent smokers can recruit the necessary resources, but when demand is perceived to be low or intermediate, these resources are not adequately recruited and performance drops. Smokers, even when abstinent, might therefore be just as capable of engaging cognitive resources as non-smokers, but simply fail to do so at intermediate levels of challenge. This interpretation may also help shed some light on inconsistencies in the literature. 
Subtle difference in the design of the tasks, or the recruited population (especially individual differences in cognitive capacity), may cause inhibitory control (Nogo, Incongruent) conditions to be perceived either as intermediately demanding (where one would expect group differences) or as very demanding (where these differences are not expected).

Imaging results showed lower right $\mathrm{Al}$ and putamen activity in smokers during the successful withholding of an inappropriate response and decreased dACC/pre-SMA activity in the presence of a nicotine patch. Reduced engagement of dependent smokers' frontal and striatal task-relevant brain areas is in line with neuroimaging studies on nicotine dependence $[21,30]$ and other substance use disorders [28]. Interestingly, the right Al has been specifically implicated in allocating cognitive resources by monitoring performance and environmental demands $[55,56]$. Moreover, structural and functional connectivity between striatum and $\mathrm{Al}$ is affected in smokers and predictive of relapse [57, 58]. Across groups, the dACC/pre-SMA, which is central to processing salience, attention, and inhibitory control in both tasks [24, 43, 44], showed less activity in the nicotine patch compared to the placebo patch condition, indicating that less recruitment of this area is needed to achieve the same performance. This may reflect the beneficial effects on attention captured by fewer omission errors and missed trials with nicotine present in both groups. Previous neuroimaging studies have similarly reported decreased dACC activity in smokers during an inhibitory control task after smoking [59] and in superior and middle frontal gyrus during an attentional task on nicotine patch [45], suggesting alleviation of withdrawal. In the current study, however, we were able to compare smokers and non-smokers and did not find a group difference. The dACC/pre-SMA downregulation by nicotine may therefore reflect increased processing efficiency rather than an alleviation of decreased efficiency. In contrast with our behavioral findings, we did not detect group-by-drug interactions in the fMRI analysis in this study. Importantly, this should not be taken to mean that there are no neural correlates of the significant attentional deficits in smokers or the improved attentional performance with nicotine in both groups. Rather, such differences were not detected using $\mathrm{fMRI}$ contrasts that captured neural activity underlying inhibition and error processing.

Our results have potential clinical implications. Manipulating the acute abstinent state in the smoker group allowed us to look at how cognitive control, as manifest using two response inhibition tasks, Go-Nogo and Flanker, and their neural signatures, are impacted by nicotine and varenicline. This is crucial to assess these drugs' ability to alleviate cognitive deficits reported during the NWS. Rather, we show that smokers' ability to pay attention to relatively easy tasks is diminished when abstaining from nicotine. Speculatively, newly abstinent smokers may be able to suppress the urge to smoke at the time and in the context they anticipate will be difficult but may be more vulnerable for relapse in contexts of lower perceived risk. It is encouraging that the deficits found were largely alleviated with the administration of a nicotine patch or (in the case of the Go-Nogo task) varenicline pill. This highlights the role smoking-cessation medication can play in supporting a quit attempt. Our results also support the use of cognitive behavioral approaches to enhance smokers' awareness of the need to remain vigilant in seemingly less challenging situations.

This study is not without limitations. First, our fMRI design was optimized to test for differences in inhibitory control, not sustained attention. In line with our behavioral results, few differences associated with successful (inhibition contrasts) and failed (error contrasts) trials were found. Our imaging tasks, particularly the Go-Nogo task, were not designed to test for differences in the less demanding control conditions, where behavioral effects did occur. We can speculate that group and session differences might have been found in the imaging results consistent with the behavioral findings. Second, there is a chance that the modest sample size may have precluded observing a group or pharmacological effect. However, it is worth noting that our sample size is comparable or larger than those in previous studies $[4,29,30,50]$ and that the current sample showed robust behavioral and imaging effects on other tasks, such as probabilistic reversal learning [39], the monetary incentive delay task [4], and an emotional reactivity task $[3,40]$, as well as on our measures of attention. Therefore, even if specific effects on inhibition were missed, these would likely have been very subtle.

Future investigations should further investigate smokers' inability to allocate cognitive and/or attentional resources at lower or intermediate levels of difficulty using fMRI designs specifically tailored to detect attentional and inhibitory effects. Moreover, effects on sustained attention, putatively mediated through nicotinic effects on norepinephrine signaling, should be factored into these designs, so that the contributions of these distinct effects might be teased apart.

\section{FUNDING AND DISCLOSURE}

This work was sponsored by the National Institute on Drug Abuse, Intramural Research Program, National Institutes of Health, US Department of Health and Human Services. EL is supported by the Flemish Fund for Scientific Research (FWO) grant FWO16/PG3/032. MTS was in part supported by National Institute on Drug Abuse grant K01DA037819, National Institute on Drug Abuse grant R01DA041353, and National Institute on Minority Health and Health Disparities grant U54MD01239 (sub-project 5378). The authors declare that there are no conflict of interest to disclose.

\section{ADDITIONAL INFORMATION}

Supplementary Information accompanies this paper at (https://doi.org/10.1038/ s41386-020-0623-1)

Publisher's note Springer Nature remains neutral with regard to jurisdictional claims in published maps and institutional affiliations.

\section{REFERENCES}

1. Center for Disease Control. Quitting smoking among adults-United States, 2001-2010. MMWR Weekly. 2014;60:1513-19.

2. Powell J, Dawkins L, West R, Powell J, Pickering A. Relapse to smoking during unaided cessation: clinical, cognitive and motivational predictors. Psychopharmacology (Berl). 2010;212:537-49. https://doi.org/10.1007/s00213-010-1975-8

3. Sutherland MT, Carroll AJ, Salmeron BJ, Ross TJ, Hong LE, Stein Ea. Downregulation of amygdala and insula functional circuits by varenicline and nicotine in abstinent cigarette smokers. Biol Psychiatry. 2013;74:1-9. https://doi.org/ 10.1016/j.biopsych.2013.01.035

4. Fedota JR, Sutherland MT, Salmeron BJ, Ross TJ, Hong LE, Stein EA. Reward anticipation is differentially modulated by varenicline and nicotine in smokers. Neuropsychopharmacology. 2015;40:2038-46. https://doi.org/10.1038/npp.2015.54

5. Rose EJ, et al. Acute nicotine differentially impacts anticipatory valence- and magnitude-related striatal activity. Biol Psychiatry. 2013;73:280-8. https://doi.org/ 10.1016/j.biopsych.2012.06.034

6. Rose EJ, et al. Chronic exposure to nicotine is associated with reduced rewardrelated activity in the striatum but not the midbrain. Biol Psychiatry. 2012;71:206-13. https://doi.org/10.1016/j.biopsych.2011.09.013

7. Ashare $\mathrm{RL}$, Falcone $\mathrm{M}$, Lerman $\mathrm{C}$. Cognitive function during nicotine withdrawal: Implications for nicotine dependence treatment. Neuropharmacology. 2014;76:581-91. https://doi.org/10.1016/j.neuropharm.2013.04.034

8. Ernst $M$, et al. Effect of nicotine on brain activation during performance of a working memory task. Proc Natl Acad Sci USA. 2001;98:4728-33.

9. Goldstein RZ, Volkow ND. Drug addiction and its underlying neurobiological basis: neuroimaging evidence for the involvement of the frontal cortex. Am J Psychiatry. 2002;159:1642-52.

10. Volkow ND, Koob GF, McLellan AT. Neurobiologic advances from the brain disease model of addiction. N Engl J Med. 2016;374:363-71. https://doi.org/10.1056/ NEJMra1511480

11. Benowitz NL. Nicotine addiction. N Engl J Med. 2010;362:2295-303. https://doi. org/10.1056/NEJMra0809890 
12. Lesage E, Stein EA. Networks associated with reward. In: Pfaff DW, ND Volkow ND, editors. Neuroscience in the 21st century. New York, NY: Springer New York; 2016. pp. 1-27.

13. Hahn B. Nicotinic receptors and attention. In: Balfour DJK, Munafò MR, editors. The neurobiology and genetics of nicotine and tobacco. Cham: Springer International Publishing; 2015. pp. 103-35.

14. Kalivas PW. The glutamate homeostasis hypothesis of addiction. Nat Rev Neurosci. 2009;10:561-72. https://doi.org/10.1038/nrn2515

15. Koob GF, Volkow ND. Neurocircuitry of addiction. Neuropsychopharmacology. 2010;35:217-38.

16. Rollema H, Coe JW, Chambers LK, Hurst RS, Stahl SM, Williams KE. Rationale, pharmacology and clinical efficacy of partial agonists of alpha4beta2 nACh receptors for smoking cessation. Trends Pharmacol Sci. 2007;28:316-25. https:// doi.org/10.1016/j.tips.2007.05.003

17. Atzori G, Lemmonds CA, Kotler ML, Durcan MJ, Boyle J. Efficacy of a nicotine (4 mg)containing lozenge on the cognitive impairment of nicotine withdrawal. J Clin Psychopharmacol. 2008;28:667-74. https://doi.org/10.1097/JCP.0b013e31818c9bb8

18. Cahill K, Lindson-Hawley N, Thomas KH, Fanshawe TR, Lancaster T. Nicotine receptor partial agonists for smoking cessation. Cochrane Database Syst Rev. 2016. https://doi.org/10.1002/14651858.CD006103.pub7

19. Hartmann-Boyce J, Chepkin SC, Ye W, Bullen C, Lancaster T. Nicotine replacement therapy versus control for smoking cessation. Cochrane Database Syst Rev. 2018. https://doi.org/10.1002/14651858.CD000146.pub5

20. Eriksen BA, Eriksen CW. Effects of noise letters upon the identification of a target letter in a nonsearch task. Percept Psychophys. 1974;16:143-9. https://doi.org/ 10.3758/BF03203267

21. Luijten M, Machielsen M, Veltman D, Hester R, de Haan L, Franken I. Systematic review of ERP and fMRI studies investigating inhibitory control and error processing in people. J Psychiatry Neurosci. 2014;39:149-69. https://doi.org/10.1503/ jpn.130052

22. Smith JL, Mattick RP, Jamadar SD, Iredale JM. Deficits in behavioural inhibition in substance abuse and addiction: a meta-analysis. Drug Alcohol Depend. 2014;145:1-33. https://doi.org/10.1016/j.drugalcdep.2014.08.009

23. Cieslik EC, Mueller VI, Eickhoff CR, Langner R, Eickhoff SB. Three key regions for supervisory attentional control: Evidence from neuroimaging meta-analyses. Neurosci Biobehav Rev. 2015;48:22-34. https://doi.org/10.1016/j.neubiorev.2014.11.003

24. Simmonds DJ, Pekar JJ, Mostofsky SH. Meta-analysis of Go/No-go tasks demonstrating that $\mathrm{fMRl}$ activation associated with response inhibition is taskdependent. Neuropsychologia. 2008;46:224-32. https://doi.org/10.1016/j. neuropsychologia.2007.07.015

25. Zhang R, Geng X, Lee TMC. Large-scale functional neural network correlates of response inhibition: an fMRI meta-analysis. Brain Struct Funct. 2017;222:3973-90. https://doi.org/10.1007/s00429-017-1443-x

26. Luijten $M$, et al. The role of dopamine in inhibitory control in smokers and nonsmokers: a pharmacological fMRI study. Eur Neuropsychopharmacol. 2013:23:1247-56. https://doi.org/10.1016/j.euroneuro.2012.10.017

27. Koob GF, Volkow ND. Neurobiology of addiction: a neurocircuitry analysis. Lancet Psychiatry. 2016;3:760-73. https://doi.org/10.1016/S2215-0366(16)00104-8

28. Kaufman JN, Ross TJ, Stein EA, Garavan H. Cingulate hypoactivity in cocaine users during a GO-NOGO task as revealed by event-related functional magnetic resonance imaging. J Neurosci. 2003;23:7839-43.

29. Luijten $M$, Littel $M$, Franken IHA. Deficits in inhibitory control in smokers during a Go/NoGo task: an investigation using event-related brain potentials. PLoS ONE. 2011;6:e18898. https://doi.org/10.1371/journal.pone.0018898

30. Nestor L, McCabe E, Jones J, Clancy L, Garavan H. Differences in 'bottom-up' and 'top-down' neural activity in current and former cigarette smokers: evidence for neural substrates which may promote nicotine abstinence through increased cognitive control. Neuroimage. 2011;56:2258-75. https://doi.org/10.1016/j. neuroimage.2011.03.054

31. Buzzell GA, Fedota JR, Roberts DM, McDonald CG. The N2 ERP component as an index of impaired cognitive control in smokers. Neurosci Lett. 2014;563:61-5. https://doi.org/10.1016/j.neulet.2014.01.030

32. Carim-Todd L, Mitchell SH, Oken BS. Impulsivity and stress response in nondependent smokers (tobacco chippers) in comparison to heavy smokers and nonsmokers. Nicotine Tob Res. 2016;18:547-56. https://doi.org/10.1093/ntr/ ntv210

33. Barr RS, et al. The effects of transdermal nicotine on cognition in nonsmokers with schizophrenia and nonpsychiatric controls. Neuropsychopharmacology. 2008;33:480-90. https://doi.org/10.1038/sj.npp.1301423

34. Valentine G, Sofuoglu M. Cognitive effects of nicotine: recent progress. Curr Neuropharmacol. 2018;16:403-14. https://doi.org/10.2174/1570159X15666171103152136

35. Foulds J, Stapleton J, Swettenham J, Bell N, McSorley K, Russell MAH. Cognitive performance effects of subcutaneous nicotine in smokers and never-smokers. Psychopharmacology (Berl). 1996;127:31-8. https://doi.org/10.1007/BF02805972
36. Heishman SJ, Kleykamp BA, Singleton EG. Meta-analysis of the acute effects of nicotine and smoking on human performance. Psychopharmacology (Berl). 2010;210:453-69. https://doi.org/10.1007/s00213-010-1848-1

37. Ettinger $U$, et al. Effects of nicotine on response inhibition and interference control. Psychopharmacology (Berl). 2017;234:1093-111. https://doi.org/10.1007/ s00213-017-4542-8

38. Zelle SL, Gates KM, Fiez JA, Sayette MA, Wilson SJ. The first day is always the hardest: functional connectivity during cue exposure and the ability to resist smoking in the initial hours of a quit attempt. Neurolmage. 2017;151:24-32. https://doi.org/10.1016/j.neuroimage.2016.03.015

39. Lesage E, Aronson SE, Sutherland MT, Ross TJ, Salmeron BJ, Stein EA. Neural signatures of cognitive flexibility and reward sensitivity following nicotinic receptor stimulation in dependent smokers: a randomized trial. JAMA Psychiatry. 2017;74:632-40. https://doi.org/10.1001/jamapsychiatry.2017.0400

40. Carroll AJ, Sutherland MT, Salmeron BJ, Ross TJ, Stein EA. Greater externalizing personality traits predict less error-related insula and anterior cingulate cortex activity in acutely abstinent cigarette smokers. Addict Biol. 2015;20:377-89. https://doi.org/10.1111/adb.12118

41. Cox RW. AFNI: software for analysis and visualization of functional magnetic resonance neuroimages. Comput Biomed Res Int J. 1996;29:162-73.

42. Afyouni S, Nichols TE. Insight and inference for DVARS. Neuroimage. 172:291-312. https://doi.org/10.1016/j.neuroimage.2017.12.098

43. Seeley WW, et al. Dissociable intrinsic connectivity networks for salience processing and executive control. J Neurosci. 2007;27:2349-56. https://doi.org/ 10.1523/JNEUROSCI.5587-06.2007

44. Xu M, Xu G, Yang $Y$. Neural systems underlying emotional and non-emotional interference processing: an ALE meta-analysis of functional neuroimaging studies. Front Behav Neurosci. 2016. https://doi.org/10.3389/fnbeh.2016.00220

45. Hahn B, Ross TJ, Wolkenberg FA, Shakleya DM, Huestis MA, Stein EA. Performance effects of nicotine during selective attention, divided attention, and simple stimulus detection: an fMRI study. Cereb Cortex. 2009;19:1990-2000. https://doi. org/10.1093/cercor/bhn226

46. Fedota $J R$, et al. Nicotine abstinence influences the calculation of salience in discrete insular circuits. Biol Psychiatry Cogn Neurosci Neuroimaging. 2018;3:150-9. https://doi.org/10.1016/j.bpsc.2017.09.010

47. Grundey J, Amu R, Ambrus GG, Batsikadze G, Paulus W, Nitsche MA. "Double dissociation of working memory and attentional processes in smokers and nonsmokers with and without nicotine,". Psychopharmacology (Berl). 2015;232:2491-501. https://doi.org/10.1007/s00213-015-3880-7

48. Lawrence NS, Ross TJ, Stein EA. Cognitive mechanisms of nicotine on visual attention. Neuron. 2002;36:539-48. https://doi.org/10.1016/S0896-6273(02) 01004-8

49. Sutherland MT, Carroll AJ, Salmeron BJ, Ross TJ, Hong LE, Stein EA. Individual differences in amygdala reactivity following nicotinic receptor stimulation in abstinent smokers. Neuroimage. 2013;66:585-93. https://doi.org/10.1016/j. neuroimage.2012.10.043

50. Kozink RV, Kollins SH, McClernon FJ. Smoking withdrawal modulates right inferior frontal cortex but not presupplementary motor area activation during inhibitory control. Neuropsychopharmacology. 2010;35:2600-6. https://doi.org/10.1038/ npp.2010.154

51. Weywadt CR, Kiehl KA, Claus ED. Neural correlates of response inhibition in current and former smokers. Behav Brain Res. 2017;319:207-18. https://doi.org/ 10.1016/j.bbr.2016.11.030

52. Rass O, Fridberg DJ, O'Donnell BF. Neural correlates of performance monitoring in daily and intermittent smokers. Clin Neurophysiol. 2014;125:1417-26. https:// doi.org/10.1016/j.clinph.2013.12.001

53. Fedota JR, Matous AL, Salmeron BJ, Gu H, Ross TJ, Stein EA. Insula demonstrates a non-linear response to varying demand for cognitive control and weaker resting connectivity with the executive control network in smokers. Neuropsychopharmacology. 2016;41:2557-65. https://doi.org/10.1038/npp.2016.62

54. Evans DE, Craig C, Oliver JA, Drobes DJ. The smoking N-back: a measure of biased cue processing at varying levels of cognitive load. Nicotine Tob Res. 2011;13:88-93. https://doi.org/10.1093/ntr/ntq214

55. Dosenbach NUF, et al. Distinct brain networks for adaptive and stable task control in humans. Proc Natl Acad Sci USA. 2007;104:11073-8. https://doi.org/ 10.1073/pnas.0704320104

56. Eckert MA, et al. At the heart of the ventral attention system: the right anterior insula. Hum Brain Mapp. 2009;30:2530-41. https://doi.org/10.1002/hbm.20688

57. Yuan K, et al. Striato-cortical tracts predict 12-h abstinence-induced lapse in smokers. Neuropsychopharmacology. 2018;43:2452-8. https://doi.org/10.1038/ s41386-018-0182-x

58. $\mathrm{Bi} Y$, et al. Altered resting state functional connectivity of anterior insula in young smokers. Brain Imaging Behav. 2017;11:155-65. https://doi.org/10.1007/s11682016-9511-z 
Nicotine dependence (trait) and acute nicotinic stimulation (state)...

E Lesage et al.

59. Azizian A, Nestor LJ, Payer D, Monterosso JR, Brody AL, London ED. Smoking reduces conflict-related anterior cingulate activity in abstinent cigarette smokers performing a stroop task. Neuropsychopharmacology. 2010;35:775-82. https:// doi.org/10.1038/npp.2009.186

60. Nestor L, McCabe E, Jones J, Clancy L, Garavan H. Differences in 'bottom-up' and 'top-down' neural activity in current and former cigarette smokers: evidence for neural substrates which may promote nicotine abstinence through increased cognitive control. Neurolmage. 2011;56:2258-75. https://doi.org/10.1016/j. neuroimage.2011.03.054

61. Hester R, Fassbender C, Garavan H. Individual differences in error processing: review and reanalysis of three event-related fMRI studies using the GO/NOGO task. Cereb Cortex. 2004;14:986-94. https://doi.org/10.1093/cercor/bhh059 